\title{
Architectures down to nano
}

\author{
Nanoarchitecture design of materials offers great opportunities to achieve unusual properties, but requires \\ fabrication techniques that ensure sufficient resolution and material quality.
}

A rchitected materials, consisting of periodic unit cells, can demonstrate exotic properties by engineering the structure and topological arrangements of the unit cells. Biological systems, whose structures have evolved over millions of years to reach optimized functions, offer abundant inspiration for structural design. Atomic lattices and microstructures of crystals have also been used as reference models. Alternatively, reverse engineering can identify optimal structures by starting from the targeted functions and using computational methods.

Equally important to design advances is the development of fabrication techniques that can have precise control of the chemical composition, material quality and geometry. This can be extremely challenging for complex structures that span multiple length scales, down to nanosized features. Nanoarchitected materials harness beneficial size effects on the mechanical and physical properties combined with a large surface area. However, materials that can be shaped into nanoarchitectures are still technologically constrained, especially for non-polymer materials. In this issue of Nature Materials, we present several studies showing advances in the fabrication of carbon-, ceramic- and metal-based lattice structures with nanoscale features and their peculiar properties operating beyond conventional regimes.

Additive manufacturing (AM) has been commonly used to print hierarchical structures. Most AM processes, however, can only reach micrometre resolution based on photocuring of polymeric materials. Post-processing such as pyrolysis, sintering, coating and etching has to be integrated to obtain nanostructured metals, ceramics or composites, and to remove the polymer templates.

For instance, pyrolysis of printed polymeric microlattices leads to carbon nanolattices with high strength-to-weight ratio. Under supersonic impact, these carbon nanolattices exhibit extreme energy dissipation due to their capability to propagate compaction shocks, as shown by Carlos Portela and colleagues in their Article. However, volume shrinkage due to pyrolysis does not allow carbon nanolattices to reach the ultralow-density regime $\left(10 \mathrm{mg} \mathrm{cm}^{-3}\right)$. To further reduce the density, Jianchao Ye and colleagues describe in

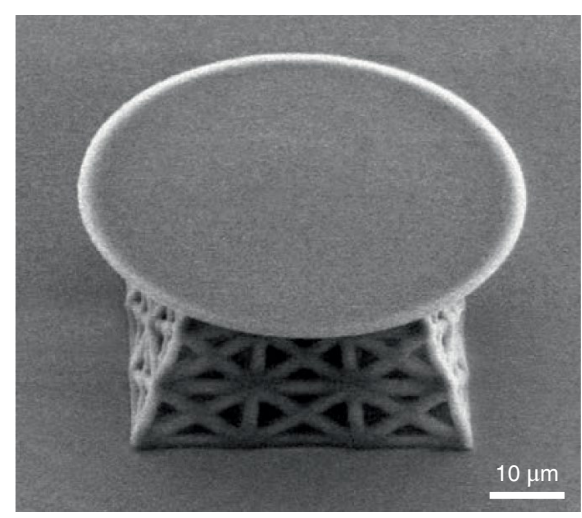

glass or crystalline polymorphs, by using a two-photon-polymerizable precursor containing a high loading of colloidal silica nanoparticles. Doping and co-doping of rare-earth elements can be directly implemented. The proof-of-concept demonstration of microtoroid whispering gallery resonators with high quality factors may be promising for biosensing and point-of-care diagnostic tests, as noted in a News and Views article by Paolo Colombo and Giorgia Franchin.

In addition to AM methods, nanolattices can be obtained through a low-cost and fast self-assembly process, with molecules or particles spontaneously organizing into an ordered structure. Such structures have often been used as templates to realize inverse opals with a variety of materials, achieved by filling the template with a precursor material, converting the precursor to the target solid, and finally removing the template. However, this process creates dense cracks within the structure that severely constrain its size and properties. Zhimin Jiang and James Pikul describe in their Article the preparation of self-standing, centimetre-scale and crack-free inverse opal nickel nanolattices by optimizing the co-assembly and electroplating processes. This enables the fabrication of samples for tensile testing, which show high tensile strength approaching the theoretical limits. As commented by Andreas Stein and Nathan Mara in a News and Views article, eliminating the cracks not only allows the development of high-strength, lightweight materials, but also the further exploration of the nuanced effects of ligament-node geometries and local composition on the mechanical behaviour of these nanolattices.

Although nanoarchitecture control provides more freedom for materials design, it does not yet apply to many types of materials, composites or hybrid structures due to the lack of manufacturing solutions guaranteeing suitable precision over multiple scales while maintaining material quality. Scalability in terms of the size of the sample and production rate is also an important issue. But advances reported in these studies suggest that there are many innovative solutions worth exploring further.

Published online: 25 October 2021 https://doi.org/10.1038/s41563-021-01147-4 